九州大学学術情報リポジトリ

Kyushu University Institutional Repository

\title{
ON A CLASS MULTISAMPLE RANK TESTS BASED ON TRIMMED SAMPLES
}

Tamura, Ryoj i

Department of Mathematics, Kumamoto University

https://doi.org/10.5109/13057

出版情報: 統計数理研究. 15 (1/2), pp.1-6, 1972-03. Research Association of Statistical Sciences

バージョン :

権利関係 : 


\title{
ON A CLASS OF MULTISAMPLE RANK TESTS BASED ON TRIMMED SAMPLES
}

\author{
By \\ Ryoji TAMURA*
}

(Received May 5, 1971)

\section{Introduction.}

Hettmansperger [5] has shown that the Mann-Whitney test has larger asymptotic relative efficiencies in Pitman's sense for some distributions with heavy tails by using the trimmed samples instead of the complete samples. Recently Tamura [8] has generalized Hettmansperger's results to Bhapkar's test [3] for the $c$-sample problem. Along the same line as [8], we shall propose a class of rank tests based on the trimmed samples and discuss in detail about Kruskal-Wallis test [6] as its special case.

Let $X_{i 1}<\cdots<X_{i n_{i}}$ be order statistics from absolutely continuous cdf $F_{i}(x)$ $=F\left(x-\theta_{i}\right), i=1, \cdots, c$ where $F(x)$ has symmetric density $f(x)$ of unknown functional form. We further assume for $0<\alpha<-\frac{1}{2}$ that $f(x)$ is continuously differentiable in some neighborhood of the unique population quantiles $b_{\alpha}$ and $b_{1-\alpha}$ of order $\alpha$ and $1-\alpha$, respectively. The hypothesis $H_{0}$, to be tested, is specified by $\theta_{1}=\cdots=\theta_{c}$ against the alternatives that not all $\theta$ 's are equal. For this problem, a class of test statistics will be proposed on the basis of only the middle $n_{i}-2 k_{i}$ random variables $X_{i k_{i+1}}<\cdots<X_{i n_{i-k}}, i=1, \cdots, c$, where $k_{i}=\left[n_{i} \alpha\right]$ denotes the largest integer not exceeding $n_{i} \alpha$. Throughout this paper, we assume that the sample size $n_{i}, i=1$, $\cdots, c$, increases in such a way that $\lim _{N \rightarrow \infty} n_{i} / N=\lambda_{i}, 0<\lambda_{i}<1$ where $N=\sum_{i=1}^{c} n_{i}$. Some definitions and assumptions are given in Section 2. In Section 3, we derive the asymptotic distributions of the proposed statistics. Section 4 is concerned with the test of Kruskal-Wallis type.

\section{Definitions and assumptions.}

Let us define for $i=1, \cdots, c$

$$
T_{i}=\left(n_{i}-2 k_{i}\right)^{-1} \sum_{\beta=1}^{N-2 k} E_{\beta}^{(i)} Z_{\beta}^{(i)}, \quad k=\sum_{i=1}^{c} k_{i}
$$

where

\footnotetext{
* Department of Mathematics, Kumamoto University, Kumamoto.
} 


$$
Z_{\beta}^{\langle i\rangle}= \begin{cases}1 & \begin{array}{l}
\text { if the } \beta \text { th smallest among the combined trimmed } \\
\text { samples is from } F_{i}(x)
\end{array} \\
0 & \text { otherwise }\end{cases}
$$

and the $E_{\beta}^{(i)}$ 's are some given constants. Then we can represent $T_{i}$ by

$$
T_{i}=\int_{-\infty}^{\infty} J_{N}\left(H_{N}(x)\right) d G_{i}^{*}\left(x, n_{i}-2 k_{i}\right)
$$

where we set $J_{N}(\beta /(N-2 k+1))=E_{\beta}^{(i)}$ and $G_{i}^{*}\left(x, n_{i}-2 k_{i}\right)$ is the empirical cdf based on the $i$-th trimmed sample and

$$
H_{N}(x)=\sum_{i=1}^{c} \lambda_{i} G_{i}^{*}\left(x, n_{i}-2 k_{i}\right) .
$$

Assumption (A).

(i) $\lim _{N \rightarrow \infty} J_{N}(t)=J(t)$ exists for $0<t<1$ and is not constant.

(ii) $\int_{I_{N}}\left[J_{N}\left(H_{N}\right)-J\left(H_{N}\right)\right] d G_{i}^{*}\left(x, n_{i}-2 k_{i}\right)=o_{p}\left(N^{-12}\right), I_{N}=\left\{x ; o<H_{N}(x)<1\right\}$

(iii) $J_{N}(1)=o\left(N^{1}\right)$

(iv) $\left|d^{j} J(t) / d t^{j}\right| \leqq M(t(1-t))^{-j-\frac{1}{2}+\delta}, j=0,1,2$

and some $\delta>0$ where $M$ is a generic constant.

The form (2.2) and the assumption (A) have been dealt by Chernoff-Savage [4] and Puri [7]. Further we define for $i=1, \cdots, c$

$$
\begin{aligned}
& Y_{i 1}=n_{i}^{\frac{1}{2}}\left(X_{i k_{i+1}}-\theta_{i}-b_{\alpha}\right), \quad Y_{i 2}=n_{i}^{\frac{1}{2}}\left(X_{i n_{i}-k_{i}}-\theta_{i}-b_{1-\alpha}\right) \\
& \boldsymbol{Y}^{\prime}=\left(Y_{11}, Y_{12}, \cdots, Y_{c 1}, Y_{c 2}\right) .
\end{aligned}
$$

We here notice that the statistics $T_{i}$, given $\boldsymbol{Y}$, are the the rank statistics of ChernoffSavage type [4] based on the trimmed samples from the $\operatorname{cdf} G_{i}(x)$ with density $g_{i}(x)$,

$$
g_{i}(x)=\left\{\begin{array}{l}
f\left(x-\theta_{i}\right) /\left[F\left(b_{1-\alpha}+Y_{i 2} / n_{i}^{\frac{1}{2}}\right)-F\left(b_{\alpha}+Y_{i 1} / n_{i}^{\frac{1}{2}}\right)\right] \\
\quad \text { for } b_{\alpha}+\theta_{i}+Y_{i 1} / n_{i}^{\frac{1}{2}} \leqq x \leqq b_{1-\alpha}+\theta_{i}+Y_{i 2} / n_{i}^{\frac{1}{2}} \\
0 \quad \text { otherwise } .
\end{array}\right.
$$

Finally, we define for $i=1, \cdots, c$

$$
\begin{array}{ll}
R_{i}=(N-2 k)^{\frac{1}{2}}\left(T_{i}-E\left(T_{i} \mid \boldsymbol{Y}\right)\right), & \boldsymbol{R}^{\prime}=\left(R_{1}, \cdots, R_{c-1}\right) \\
W_{i}=(N-2 k)^{\frac{1}{2}}\left(T_{i}-\int_{0}^{1} J(t) d t\right), & W^{\prime}=\left(W_{1}, \cdots, W_{c-1}\right)
\end{array}
$$

where $E(* \mid \boldsymbol{Y})$ is the expected value of the statistic $*$, given $\boldsymbol{Y}$. 


\section{Asymptotic distributions.}

Now we shall consider the asymptotic distributions of the proposed statistics under the hypothesis $H_{0}$ and the following sequence of alternatives

$$
H_{N}: F_{i}(x)=F\left(x-\nu_{i} / N^{\frac{1}{2}}\right), \quad i=1, \cdots, c
$$

where not all $\nu$ 's are equal.

LEMMA 3.1. The random vector $(\boldsymbol{R}, \boldsymbol{Y})$ is asymptotically normally distributed if the assumption $(A)$ holds.

PRoof. It has been shown by Puri [7] that the random vector $\boldsymbol{R}$ has the joint normal distribution $N\left(0, \Sigma^{(1)}\right)$, given $Y$, if the assumption (A) holds where $\Sigma^{(1)}=$ $\left\|\sigma_{i j}^{(1)}\right\| i, j=1, \cdots, c-1$

$$
\sigma_{i j}^{(1)}=\lambda_{i}^{-1}\left(\delta_{i j}-\lambda_{i}\right)\left[\int_{0}^{1} J^{2}(t) d t-\left(\int_{0}^{1} J(t) d t\right)^{2}\right]
$$

where $\delta_{i j}=1$ or 0 for $i=j$ or $i \neq j$. It is also well known that $\boldsymbol{Y}$ has the asymptotic normal cdf $N(\mathbf{0}, \Omega)$ where

$$
\boldsymbol{\Omega}=\left\|\begin{array}{ccc}
\boldsymbol{\Omega}_{1} & \cdots & 0 \\
\cdots \cdots & \cdots \\
0 & \cdots & \boldsymbol{\Omega}_{1}
\end{array}\right\| \quad \boldsymbol{\Omega}_{1}=f^{-2}\left(b_{\alpha}\right)\left\|\begin{array}{cc}
\alpha(1-\alpha) & \alpha^{2} \\
\alpha^{2} & \alpha(1-\alpha)
\end{array}\right\|
$$

These facts establish the asymptotic normality of $(\boldsymbol{R}, \boldsymbol{Y})$.

THEOREM 3.1. The random vector $\boldsymbol{W}$ has the asymptotic normal distribution under the assumption $(A)$ and $J(1)<\infty, J(0)<\infty$.

PROOF. By expanding the Puri's results [7]

$$
E\left(T_{i} \mid \boldsymbol{Y}\right)=\int_{-\infty}^{\infty} J\left[\sum_{k=1}^{c} \lambda_{k} G_{k}(x)\right] d G_{i}(x)+O_{p}\left(N^{-1}\right)
$$

in a Taylor series, we get the following (3.4).

where

$$
\begin{aligned}
& E\left(T_{i} \mid \boldsymbol{Y}\right)=\int_{0}^{1} J(t) d t+\left(\nu_{i}-\bar{\nu}\right) \int_{b_{\alpha}}^{b_{1}-\alpha} J^{\prime}[(F(t)-\alpha) /(1-2 \alpha)] f(t) d F(t) \\
& \quad \div N^{\frac{1}{2}}(1-2 \alpha)^{2}+\sum_{k=1}^{c} \lambda_{k} n_{k}^{-\frac{1}{2}}\left(r_{1} Y_{k 1}-r_{2} Y_{k 2}\right)+n_{i}^{-\frac{1}{2}}\left(s_{1} Y_{i 1}-s_{2} Y_{i 2}\right)+O_{q}\left(N^{-1}\right)
\end{aligned}
$$

$$
\begin{aligned}
& r_{1}=(1-2 \alpha)^{-3} f\left(b_{\alpha}\right) \int_{b_{\alpha}}^{b_{1-\alpha}} J^{\prime}[(F(t)-\alpha /(1-2 \alpha)](F(t)-1+\alpha) d F(t) \\
& r_{2}=(1-2 \alpha)^{-3} f\left(b_{1-\alpha}\right) \int_{b_{\alpha}}^{b_{1-\alpha}} J^{\prime}[(F(t)-\alpha) /(1-2 \alpha)](F(t)-\alpha) d F(t) \\
& s_{1}=(1-2 \alpha)^{-2} f\left(b_{\alpha}\right)\left[\int_{b_{\alpha}}^{b_{1-\alpha}} J[(F(t)-\alpha) /(1-2 \alpha)] d F(t)-(1-2 \alpha) J(0)\right] \\
& s_{2}=(1-2 \alpha)^{-2} f\left(b_{1-\alpha}\right)\left[\int_{b_{\alpha}}^{b_{1-\alpha}} J[(F(t)-\alpha) /(1-2 \alpha)] d F(t)-(1-2 \alpha) J(1)\right] \\
& \bar{\nu}=\sum_{i=1}^{c} \lambda_{i} \nu_{i} .
\end{aligned}
$$


For any constant vector $\boldsymbol{a}^{\prime}=\left(a_{1}, \cdots, a_{c-1}\right)$, the scalor product $\boldsymbol{a}^{\prime} \boldsymbol{W}$, where

$$
\boldsymbol{a}^{\prime} \boldsymbol{W}=\boldsymbol{a}^{\prime} \boldsymbol{R}+(N-2 k)^{\frac{1}{2}} \sum_{i=1}^{c-1} a_{i}\left[E\left(T_{i} \mid \boldsymbol{Y}\right)-\int_{0}^{1} J(t) d t\right],
$$

becomes to a linear function of $\boldsymbol{R}$ and $\boldsymbol{Y}$ from (3.4). Thus lemma 3.1 and the theorem by Anderson [1], p. 76, lead the asymptotic normality of $\boldsymbol{a}^{\prime} \boldsymbol{W}$. The asymptotic normality of the random vector $\boldsymbol{W}$ may be established from that of $\boldsymbol{a}^{\prime} \boldsymbol{W}$ for any $\boldsymbol{a}$. We can easily see from (3.4) that $E W_{i} \sim 0$ under $H_{0}$.

Denoting the covariance matrix of $\boldsymbol{W}$ by $\Sigma$, the statistic

$$
V_{\alpha}=\boldsymbol{W}^{\prime} \Sigma^{-1} \boldsymbol{W}
$$

may be used as the test statistic for $H_{0}$. As a special case of $V_{\alpha}$, we shall, in Section 4 , discuss about the test of Kruskal-Wallis type.

\section{The test of Kruskal-Wallis type.}

The test statistic of Kruskal-Wallis type can be obtained by setting $J(t)=t$ in (3.6). In this case we rewrite the statistics such as $T_{i}, W_{i}$ and etc. in the previous. sections by $T_{i}^{*}, W_{i}^{*}$ and etc.

LEMMA 4.1. The asymptotic mean vector and covariance matrix of $\boldsymbol{W}^{*}$ is given by $\mu^{\prime}=\left(\mu_{1}, \cdots, \mu_{c-1}\right)$ and $\Sigma=\left\|\sigma_{i j}\right\|$ where

$$
\begin{aligned}
& \mu_{i}=\left(\nu_{i}-\overline{\mathcal{L}}\right) \int_{b_{\alpha}}^{\delta_{1}-\alpha} f^{2}(x) d x /(1-2 \alpha)^{3 / 2}, \\
& \sigma_{i j}=\left(\delta_{i j} \lambda_{i}^{-1}-1\right)(1+4 \alpha) / 12(1-2 \alpha)
\end{aligned}
$$

Proof. From the identity

$$
\begin{aligned}
E\left(T_{i} \mid \boldsymbol{Y}\right)= & \frac{1}{2}+\left(\nu_{i}-\bar{\nu}\right) \int_{b_{\alpha}}^{b_{1}-\alpha} f^{2}(x) d x / N^{\frac{1}{2}}(1-2 \alpha)^{2} \\
& +f\left(b_{\alpha}\right)\left(n_{i}{ }^{-\frac{1}{2}} Z_{i}-\sum_{k=1}^{c} \lambda_{k} n_{k}{ }^{-\frac{1}{2}} Z_{k}\right) / 2(1-2 \alpha)+O_{p}\left(N^{-1}\right) \\
Z_{j}= & Y_{j 1}+Y_{j 2}
\end{aligned}
$$

which is obtained from (3.4), we first get

Next from

$$
E W_{i}^{*}=\left(\nu_{i}-\bar{\nu}\right) \int_{b_{\alpha}}^{b_{1}-\alpha} f^{2}(x) d x /(1-2 \alpha)^{3 / 2}
$$

we get

$$
W_{i}^{*}=R_{i}^{*}+(N-2 k)^{\frac{1}{2}}\left[E\left(T_{i}^{*} \mid \boldsymbol{Y}\right)-1 / 2\right],
$$

$$
\begin{aligned}
\sigma_{i j}= & \operatorname{cov}\left(R_{i}^{*}, R_{j}^{*}\right)+(N-2 k) \operatorname{cov}\left(E\left(T_{i}^{*} \mid \boldsymbol{Y}\right), E\left(T_{j}^{*} \mid \boldsymbol{Y}\right)\right) \\
& +(N-2 k)^{\frac{1}{2}}\left[\operatorname{cov}\left(R_{i}^{*}, E\left(T_{j}^{*} \mid \boldsymbol{Y}\right)\right)+\operatorname{cov}\left(R_{j}^{*}, E\left(T_{i}^{*} \mid \boldsymbol{Y}\right)\right] .\right.
\end{aligned}
$$

Noticing the relations (3.2), (3.3), (4.3) and the following 
we can get

$$
\begin{aligned}
\operatorname{cov}\left(R_{i}^{*}, E\left(T_{j}^{*} \mid \boldsymbol{Y}\right)\right) & =E_{\boldsymbol{Y}}\left[\left\{E\left(T_{j}^{*} \mid \boldsymbol{Y}\right)-E\left(T_{j}^{*}\right)\right\} E\left(R_{i}^{*} \mid \boldsymbol{Y}\right)\right] \\
& =0,
\end{aligned}
$$

$$
\begin{aligned}
\sigma_{i j}= & \left(\hat{o}_{i j}-\lambda_{i}\right) / 12 \lambda_{i}+f^{2}\left(b_{\alpha}\right) E\left[\left(\lambda_{i}^{-\frac{1}{2}} Z_{i}-\sum_{k=1}^{c} \lambda_{k}{ }^{-\frac{1}{2}} Z_{k}\right)\left(\lambda_{j}-\frac{1}{2} Z_{j} \sum_{k=1}^{c} \lambda_{k}^{-\frac{1}{2}} Z_{k}\right)\right] \\
& \quad \div 4(1-2 \alpha) \\
= & \left(\delta_{i j}-\lambda_{i}\right)(1 \div 4 \alpha) / 12 \lambda_{i}(1-2 \alpha) .
\end{aligned}
$$

Under $H_{0}$ we get $\mu=0$ by setting all $\nu_{i}=0$.

THEOREM 4.1. The test statistic

$$
V_{c}^{*}=12(1-2 \alpha)(1+4 \alpha)^{-1} N^{-1} \sum_{i=1}^{c} n_{i} W_{i}^{* 2}
$$

is asymptotically distributed as $x_{t-1}^{2}$ with $c-1$ degree of freedom under $H_{0}$ and as noncentral $x_{c-1}^{2}\left(\hat{\partial}_{a}\right)$ with $c-1$ degree of freedom and the noncentrality parameter $\delta_{\alpha}$ under $H_{N}$ where

$$
\delta_{r}=12 \sum_{i=1}^{c} \lambda_{i}\left(\nu_{i}-\bar{\Sigma}\right)^{2}\left(\int_{h_{\alpha}}^{b_{1}-\alpha} f^{2}(x) d x\right)^{2} /(1-2 \alpha)^{2}(1+4 \alpha) .
$$

PROOF. It follows from Theorem 3.1 and Lemma 4.1 that $\boldsymbol{W}^{*}$ is asymptotically normal $N(\mathbf{0}, \Sigma)$ under $H_{0}$ and $N(\mu, \Sigma)$ under $H_{N}$. Therefore $\boldsymbol{W}^{* /} \Sigma^{-1} \boldsymbol{W}^{*}$ is asymtotically distributed as $x_{c-1}^{2}$ under $H_{0}$ and $x_{c-1}^{2}\left(\tilde{o}_{n}\right)$ under $H_{N}$ where

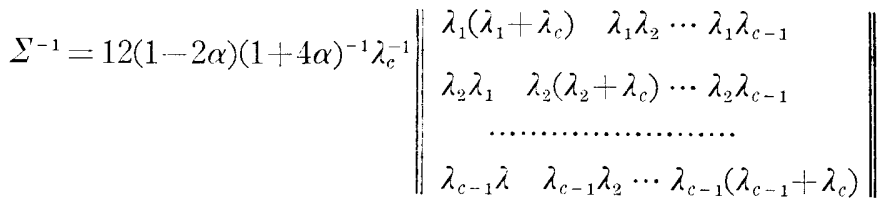

$$
\begin{aligned}
& \delta_{\alpha}=\mu^{\prime} \Sigma^{-1} \mu .
\end{aligned}
$$

Some calculations show that

$$
\boldsymbol{W}^{* /} \Sigma^{-1} \boldsymbol{W}^{*}=12(1-2 \alpha)(1+4 \alpha)^{-1} \sum_{i=1}^{c} \lambda_{i} W_{i}^{* 3} .
$$

and

$$
\mu^{\prime} \Sigma^{-1} \mu=12 \sum_{i=1}^{c} \lambda_{i}\left(\nu_{i}-\bar{\Sigma}\right)^{2}\left(\int_{b_{c e}}^{b_{1}-\alpha} f^{2}(x) d x\right)^{2} /(1-2 \alpha)^{2}(1+4 \alpha) .
$$

We here notice that Kruskal-Wallis's test may be denoted by $V_{0}^{*}$.

It has been shown by Andrews [2] that the Pitman efficiency $e_{\alpha}$ of the $V_{\alpha}^{*}$ test respective to the $V_{0}^{*}$ test is given by the ratio of the noncentrality parameters in the asymptotic $x_{c-1}^{2}$ distributions of their test statistics. From (4.5), we have

$$
e_{\alpha}=\left(\int_{b_{\alpha}}^{h_{1}-\alpha} f^{2}(x) d x\right)^{2} /(1+4 \alpha)(1-2 \alpha)^{2}\left(\int_{-\infty}^{\infty} f^{2}(x) d x\right)^{2}
$$

Lastly we give the numerical values of $e_{r}$ for some distributions with heavier tails than the normal distribution. 
Table of $e_{\alpha}$.

\begin{tabular}{l|rrrrrr}
\hline \multicolumn{1}{c|}{$\alpha$} & .1 & .2 & .25 & .3 & .35 & .4 \\
\hline Logistic & .99 & .97 & .95 & .92 & .88 & .84 \\
D. Exp. & 1.03 & 1.09 & 1.13 & 1.16 & 1.20 & 1.25 \\
Cauchy & 1.09 & 1.26 & 1.34 & 1.40 & 1.43 & 1.44 \\
\hline
\end{tabular}

\section{References}

[1] Anderson, T.W. (1958) Introduction of multivariate statistical analysis. Wiley, New York.

[2] ANDREws, F.C. (1954) Asymptotic behavior of some rank tests for analysis of variance, Ann. Math. Statist., 25 724-736.

[3] Bhapkar, V.P. (1961) A nonparametric test for the problem of several samples, Ann. Math. Statist., 32 1108-1117.

[4] Chernoff, H. and Savage, I.R. (1958) Asymptotic normality and efficiency of certain non-parametric test statistics, Ann. Math. Statist., 29 972-994.

[5] Hettmansperger, T.P. (1968) On the trimmed Mann-Whitney statistic, Ann. Math. Statist. 39 1610-1614.

[6] KRUSKAL, W.H. and Wallis, W.A. (1952) Use of ranks in one-criterion varance analysis, J.A.S. A., 47 583-621.

[7] Puri, M.L. (1964) Asymptotic efficiency of a class of c-sample tests, Ann. Math. Statist., 35 102-121.

[8] Tamura, R. (1971) On a c-sample test based on trimmed samples, Ann. Math. Statist., $421455-1460$. 